\title{
Efficacy and safety of sequential neoadjuvant chemotherapy and short-course radiation therapy followed by delayed surgery in locally advanced rectal cancer: a single-arm phase II clinical trial with subgroup analysis between the older and young patients
}

\author{
Alimohammad Bananzadeh ${ }^{1}$, Ali Akbar Hafezi ${ }^{2}$, NamPhong Nguyen ${ }^{3}$, Shapour Omidvari ${ }^{4}$, Ahmad Mosalaei ${ }^{4}$, \\ Niloofar Ahmadloo ${ }^{4}$, Mansour Ansari ${ }^{4}$, Mohammad Mohammadianpanah ${ }^{1}$ \\ ${ }^{1}$ Colorectal Research Center, Department of Colorectal Surgery, School of Medicine, Shiraz University of Medical Sciences, Shiraz, Iran \\ ${ }^{2}$ Department of Radiation Oncology, School of Medicine, Shiraz University of Medical Sciences, Shiraz, Iran \\ ${ }^{3}$ Department of Radiation Oncology, Howard University Hospital, Washington, DC, USA \\ ${ }^{4}$ Breast Diseases Research Center, School of Medicine, Shiraz University of Medical Sciences, Shiraz, Iran
}

Received: June 24, 2021

Revised: July 15, 2021

Accepted: July 22, 2021

\section{Correspondence:}

Mohammad Mohammadianpanah

Colorectal Research Center,

Department of Colorectal Surgery,

School of Medicine, Shiraz University

of Medical Sciences, Shiraz, Iran

Tel: +987136474320

E-mail:mohpanah@gmail.com

ORCID:

https://orcid.org/0000-0001-9391-8977
Purpose: This study was performed to investigate the efficacy and safety of short-course radiation therapy (SCRT) and sequential chemotherapy followed by delayed surgery in locally advancer rectal cancer with subgroup analysis between the older and young patients.

Materials and Methods: In this single-arm phase II clinical trial, eligible patients with locally advanced rectal cancer (T3-4 and/or N1-2) were enrolled. All the patients received a median three sequential cycles of neoadjuvant CAPEOX (capecitabine + oxaliplatin) chemotherapy. A total dose of 25 Gy in five fractions during 1 week was prescribed to the gross tumor and regional lymph nodes. Surgery was performed about 8 weeks following radiotherapy. Pathologic complete response rate (pCR) and grade 3-4 toxicity were compared between older patients ( $\geq 65$ years) and younger patients $(<65$ years).

Results: Ninety-six patients with locally advanced rectal cancer were enrolled. There were 32 older patients and 64 younger patients. Overall pCR was $20.8 \%$ for all the patients. Older patients achieved similar $p C R$ rate ( $18.7 \%$ vs. $21.8 ; p=0.795)$ compared to younger patients. There was no statistically significance in terms of the tumor and the node downstaging or treatment-related toxicity between older patients and younger ones; however, the rate of sphincter-saving surgery was significantly more frequent in younger patients ( $73 \%$ vs. $53 \% ; p=0.047$ ) compared to older ones. All treatment-related toxicities were manageable and tolerable among older patients.

Conclusion: Neoadjuvant SCRT and sequential chemotherapy followed by delayed surgery was safe and effective in older patients compared to young patients with locally advanced rectal cancer.

Keywords: Rectal neoplasms, Neoadjuvant therapy, Chemoradiotherapy, Colorectal surgery, Response Evaluation Criteria in Solid Tumors

Copyright (C) 2021 The Korean Society for Radiation Oncology

This is an Open Access article distributed under the terms of the Creative Commons Attribution Non-Commercial License (http://creativecommons.org/licenses/by-nc/4.0/) which permits unrestricted non-commercial use, distribution, and reproduction in any medium, provided the original work is properly cited. 


\section{Introduction}

Rectal cancer is one of the most common cancers of the gastrointestinal tract with a high recurrence and mortality rate. Survival rates for patients with rectal cancer have improved over the past three decades due to early detection in the early stages, a reduction in postoperative mortality and successful treatment in stages I and II of the disease [1]. However, locally advanced rectal cancer remains a challenge because of the high recurrence rate following surgery [2].

Neoadjuvant chemoradiation has been introduced and proven effective to reduce locoregional recurrence of those patients in randomized trials [3]. Radiotherapy can also be used to relieve local tumor symptoms [4]. Many protocols of preoperative chemoradiation were investigated comparing a short course of radiotherapy over a week to a longer course over five weeks [4-12]. The shorter course of radiotherapy was attractive especially for older patients with cancer because it reduced the need for daily transportation and hospital visits especially in light of the coronavirus disease 2019 (COVID-19) pandemic. Older patients with cancer are at high risk of dying if infected by the virus [13]. However, the safety and efficacy of this shorter course of radiotherapy has not been investi- gated in older patients with locally advanced cancer. Thus, we conducted this prospective study to investigate the feasibility of shortcourse radiation therapy (SCRT) in combination with neoadjuvant preoperative chemotherapy for those patients.

\section{Materials and Methods}

\section{Patient eligibility}

In this phase II prospective clinical trial, eligible patients with locally advanced rectal cancer (T3-4 and/or N1-2) were enrolled between 2019 and 2020. Inclusion criteria included all adult patients ( $\geq 18$ years) with newly diagnosed rectal cancer (up to $15 \mathrm{~cm}$ distance from the anal verge); histologically confirmed adenocarcinoma; clinical stage of T3 or T4 and/or N1-2; the Eastern Cooperative Oncology Group (ECOG) performance status <2; adequate bone marrow, hepatic, and renal functions.

Exclusion criteria included all patients with pregnancy; other primary malignancies; any type of distant metastasis; serious medical conditions (including severe cardiovascular, renal and/or hepatic failure); previous pelvic radiation therapy; hypersensitivity reaction to fluorouracil, oxaliplatin or capecitabine; patients' refusal to participate in the study (Fig. 1).

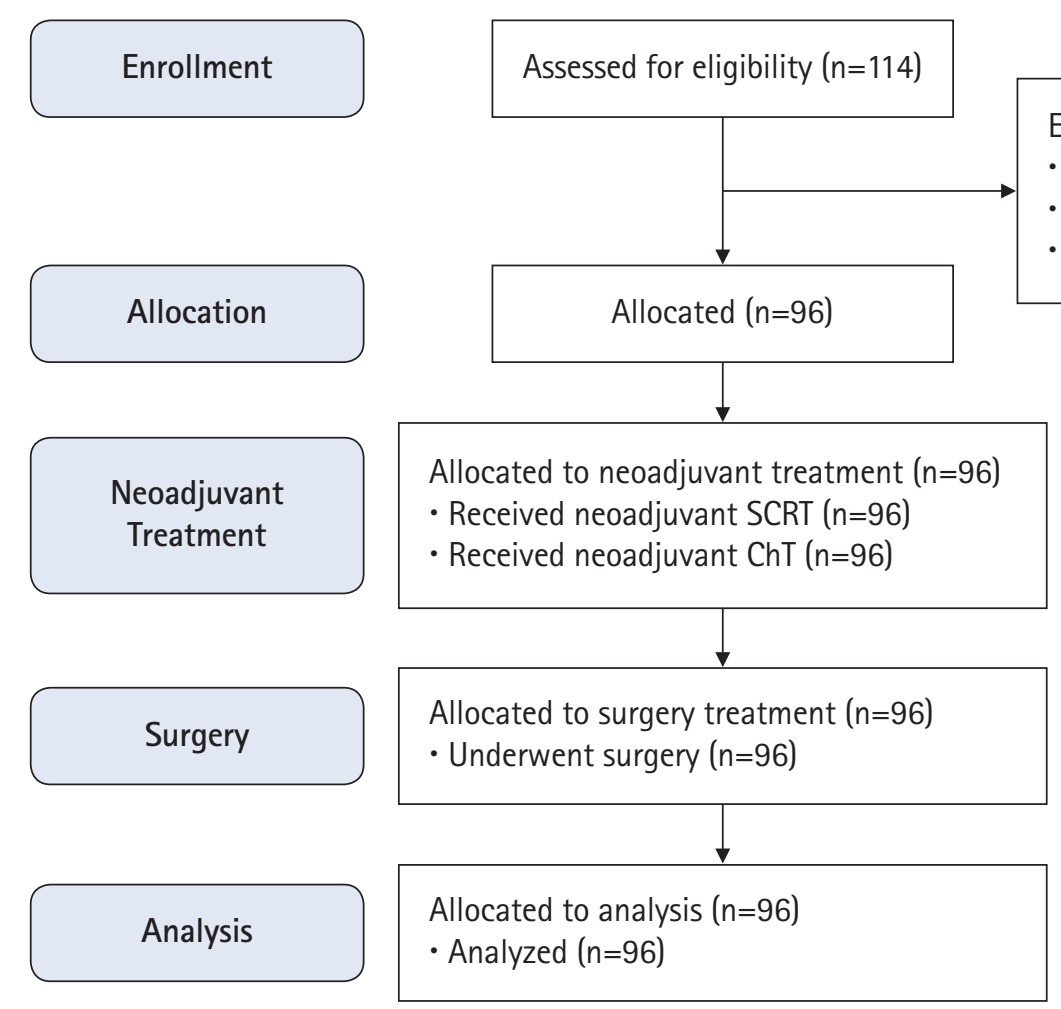

Excluded $(n=18)$

- Not meeting inclusion criteria $(n=10)$

- Declined to participate $(n=7)$

- Previous pelvic radiotherapy $(n=1)$

Fig. 1. CONSORT flow chart study. SCRT, short-course radiation therapy; ChT, chemotheraphy. 


\section{Preliminary evaluation}

Demographic and clinical characteristics of eligible patients such as age, sex, medical and surgical history, and physical examination were recorded using a data collection form. All participants were asked for any history of cardiac, liver, or kidney dysfunction and allergic reactions to the drug. All the patients underwent colonoscopy by a gastroenterologist and the site and characteristics of the tumor and its distance from the anal verge were assessed. The diagnosis of rectal adenocarcinoma was verified by pathologic examination of the biopsy specimen. Before starting treatment, all the patients underwent digital rectal examination (DRE), abdominal and pelvic computed tomography (CT) scans, and pelvic magnetic resonance imaging (MRI). Clinical staging of the tumor was performed using TNM classification according to the 8th edition of the American Joint Committee on Cancer (AJCC) staging system and completed based on MRI and CT scan images. Performance status was assessed according to ECOG criteria. Complete blood cell count, liver function test, renal function test, electrolytes, and carcinoembryonic antigen was recorded.

\section{Treatment}

All the patients received a cycle of neoadjuvant chemotherapy before SCRT and two cycles of chemotherapy after SCRT and before surgery. Based on our previous study and literature finding [14], induction chemotherapy can shrink the tumor and alleviate tumor-related symptoms such as pain and bleeding; therefore, this strategy can enhance tumor response and facilitate patients' compliance. Chemotherapy was administered with either the CAPEOX (capecitabine + oxaliplatin) regimen $(80 \%)$ or oral capecitabine alone (20\%). The CAPEOX regimen consisted of oxaliplatin $130 \mathrm{mg} /$ $\mathrm{m}^{2}$ (day 1) followed by oral capecitabine $1,000 \mathrm{mg} / \mathrm{m}^{2}$ PO bid (day 1-14). During COVID-19 pandemic, according to the European Society for Medical Oncology (ESMO) guideline adapted treatment recommendation in COVID-19 era, we had to do some modification in chemotherapy regimen to decline the risk of coronavirus infection and its morbidity and mortality. Therefore, most participant particularly older patients received capecitabine alone rather than CAPEOX regimen. Three weeks after the day 1 of the first chemotherapy cycle, all the patients underwent SCRT. Subsequently, 2 weeks after the completion of SCRT, the patients received two more cycles of chemotherapy with the same regimen. Three weeks later, they underwent surgery with an average interval of 8 weeks from the end of neoadjuvant radiation therapy. Fig. 2 shows study protocol diagram.

\section{4. short-course radiation therapy}

The patients underwent a CT scan with SOMATOM Definition AS
Siemens scanner (Siemens Healthcare $\mathrm{GmbH}$, Erlangen, Germany), and the images were transferred to the treatment planning system (TPS) through a DICOM network (INFINITT Healthcare, Seoul, Korea). The target volumes were contoured based on the RTOG anorectal contouring atlas [15]. Accordingly, gross tumor volume (GTV) included all primary gross diseases and all visible and suspicious perirectal and iliac nodes. Clinical target volume (CTV) included the GTV plus 1.5-2 cm margin; as well as, the entire rectum, mesorectum, and presacral space. Internal iliac nodes were included in the CTV for all cases; however, external iliac nodes were covered for cases with T4 tumors. Planning target volume (PTV) was created by expanding CTV plus 0.5-1 cm margin. Treatment planning was performed using Prowess Panther software version 5.4 (Prowess Inc., Concord, CA, USA) and the minimum, maximum, and mean doses for target volumes as well as organs-at-risk (OARs) such as the bladder and the right and left femoral heads were evaluated on a dose-volume histogram (DVH). Subsequently, the patients underwent SCRT with a total dose of 25 Gy in 5 fraction within 5 consecutive days with three fields (one posterior field and two lateral fields) or four-field technique (anteroposterior-posteroanterior parallel opposed fields and two lateral opposed fields) for PTV coverage with 95\% isodose (according to the International Commission on Radiation Units and Measurements [ICRU] Report 62 protocol) with 18 MV photon beam using the ONCOR linear accelerator (Siemens Healthcare $\mathrm{GmbH}$ ). All the patients were treated in a single phase and no boost dose was delivered.

Acute gastrointestinal, urinary and hematologic side effects of radiation therapy were scored according the National Cancer Institute's Common Terminology Criteria for Side Effects (CTCAE) version 5.0 (grades 1 to 5) [5].

\section{Surgery}

A standard radical surgery such as low anterior resection (LAR) or abdominoperineal resection (APR) was performed for all the patients. The type of surgery (APR vs. LAR) was usually selected at diagnosis and before neoadjuvant therapies based on many factors such as tumor location, adequacy of anal sphincter function, and patient choice. However, in some patients whose tumor location was borderline (4-5 cm above anal verge), and unknown sphincter

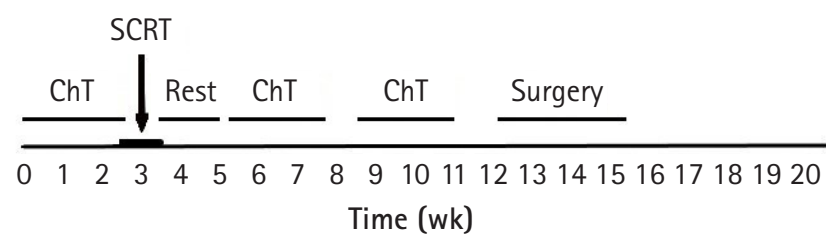

Fig. 2. Study protocol diagram. SCRT, short-course radiation therapy; ChT, chemotheraphy. 
function the choice of surgical technique was postponed until neoadjuvant treatment was completed.

\section{Pathologic response evaluation}

The postoperative pathological examination provided a microscopic description of the specimen with at least four paraffin blocks and an additional larger block. If no tumor was visible, slides were re-taken from the entire suspected area and a more accurate assessment was performed. Circumferential resection margin was defined as involved if the tumor was microscopically located less than $1 \mathrm{~mm}$ from the radial resection margin. Pathologic response was graded based on pathologist evaluation of the sample obtained from surgical resection. Downstaging was defined as any stage reduction following neoadjuvant treatments from stage III to stage 0-II, and stage II to stage 0-I confirmed in the pathological specimens [16]. PCR was defined as the absence of tumor cells at the primary site and lymph nodes. A nonresponsive tumor was defined as less than 30\% tumor shrinkage following neoadjuvant treatments [6]. In this study, we used the four AJCC tumor regression grading (TRG) classifications for evaluating. Based on this scoring system, the tumor regression is categorized as complete regression (no viable cancer cells, TRG $=0$ ), near complete regression (residual single or small groups of tumor cells, TRG $=1$ ), moderate regression (residual cancer outgrown by fibrosis, TRG $=2$ ) and finally, minimal or no regression (minimal or no tumor cells killed, $\mathrm{TRG}=3$ ) [17].

\section{Study endpoints}

The primary endpoint of the study was pCR and the secondary endpoint was adverse events. Patients were monitored weekly for acute toxicity and treatment compliance.

\section{Statistical analysis}

We calculated the study sample size based on the guidance and formula provided by Khan et al [18]. Accordingly, to improve a $10 \%$ in pCR in the present study $(\mathrm{P} 1=20 \%)$ compared to a study by Faria et al. [19] $(\mathrm{PO}=10 \%)$, and determined $\alpha=5 \%$ (exact $\alpha=$ $4.53 \%$ ) and power $=80 \%$ (exact power $=80.81 \%$ ), the number of 87 was estimated. All statistical analysis was performed using SPSS version 24 (IBM, Armonk, NY, USA). The frequency of the variables was presented by the numbers and percentages. A mean \pm standard deviation was used for continuous variables. The normality of data distribution was tested by Kolmogorov-Smirnov. Chisquare test $\left(\chi^{2}\right)$ was applied to compare the $p C R$ rate among the subgroups, and to compare the severity of treatment-related side effects at different times, Friedman test, and comparison of laboratory parameters in three times of visits, repeated-measures ANOVA were used. The significance level for all tests was considered 0.05 .

\section{Ethics statement}

The study protocol was approved by the Ethics Committee of Shiraz University of Medical Sciences in accordance with the code of ethics of the World Medical Association (Declaration of Helsinki) for experiments involving humans (No. IR.SUMS.MED.REC.1397.437). All patients signed informed consents prior to enrollment. The clinical trial was registered in the Iranian Registry of Clinical Trials (No. IRCT20190119042415N1) and all patients' data have remained confidentially.

\section{Results}

\section{Participants}

Ninety-six patients with rectal cancer underwent SCRT and chemotherapy followed by delayed surgery. Response to treatment was assessed by pathologic examination on postoperative specimens (Fig. 1).

\section{Baseline characteristics and treatment}

The median age of all patients was 59 years (range, 29 to 97 years). Sixty-four cases (66.7\%) were younger than 65 years (median, 53 years), and 32 cases (33.3\%) were 65 years or older (median, 75 years). In terms of sex, 59 subjects (64.1\%) were male, and 24 subjects (35.9\%) were female. All the patients completed their planned SCRT with similar dose, technique and scheduled. The number of chemotherapy cycles in both group was comparable; however, the CAPEOX regimen was more frequently administered in younger patients compared to older patients who were less tolerated oxaliplatin-based chemotherapy (69\% vs. 86\%; $p=0.046$ ). Table 1 summarizes patient characteristics.

Overall, APR was performed for 32 (33\%) rectal cancers and LAR in $64(67 \%)$ patients in whom the cancer was close to or involved the anal canal. Sphincter-sparing surgery was more frequent among younger patients compared to the older patients (73\% vs. $53 \% ; p=0.047)$. The reason of higher rate of APR in older patient was more due to the higher rate of impaired anal sphincter in tonometry or fecal incontinency at diagnosis, as well the patient and the surgeon preference rather than a surgical technique problem. It is believed that older patients have lower quality of life following LAR due to fecal incontinency and higher rate of LAR syndrome compared to younger patients. No serious postoperative side effects such as massive hemorrhage, infection or wound dehiscence, and leakage at the anastomosis site were observed. In terms of delay in surgery, the median time interval between SCRT and surgery was 8 weeks (range, 6 to 12 weeks). This interval was less than 8 
Table 1. Clinical characteristics of the tumor in patients with rectal cancer underwent SCRT and chemotherapy followed by delayed surgery

\begin{tabular}{|c|c|c|c|}
\hline \multirow[b]{2}{*}{ Characteristic } & \multicolumn{2}{|c|}{ Age group } & \multirow[b]{2}{*}{ p-value } \\
\hline & $\begin{array}{l}<65 y r \\
(n=64)\end{array}$ & $\begin{array}{l}\geq 65 \mathrm{yr} \\
(\mathrm{n}=32)\end{array}$ & \\
\hline Distance from AV $(\mathrm{cm})$ & $6.3 \pm 3.8$ & $6.4 \pm 3.4$ & 0.938 \\
\hline cTumor size $(\mathrm{cm})$ & $4.7 \pm 2.2$ & $5.4 \pm 2.5$ & 0.204 \\
\hline Categorized distance to AV $(\mathrm{cm})$ & & & 0.810 \\
\hline$\leq 5$ & $30(47)$ & $14(44)$ & \\
\hline $5-10$ & $26(41)$ & $15(47)$ & \\
\hline$\geq 10$ & $8(12)$ & $3(9)$ & \\
\hline cTstage & & & 0.817 \\
\hline $\mathrm{T} 2$ & $14(22)$ & $6(19)$ & \\
\hline T3 & $42(66)$ & $23(72)$ & \\
\hline $\mathrm{T} 4$ & $8(12)$ & $3(9)$ & \\
\hline cNstage & & & 0.428 \\
\hline No & $8(13)$ & $3(9)$ & \\
\hline N1 & $31(48)$ & $20(63)$ & \\
\hline $\mathrm{N} 2$ & $25(39)$ & $9(28)$ & \\
\hline cStage & & & 0.747 \\
\hline II & $8(12)$ & $3(9)$ & \\
\hline III & $56(88)$ & $29(91)$ & \\
\hline $\begin{array}{l}\text { Time interval between RT and } \\
\text { surgery (wk) }\end{array}$ & & & 0.772 \\
\hline$<8$ & $28(44)$ & $15(47)$ & \\
\hline$\geq 8$ & $36(56)$ & $17(53)$ & \\
\hline Chemotherapy regimen & & & 0.046 \\
\hline CAPEOX & $55(86)$ & $22(69)$ & \\
\hline Capecitabine & $9(14)$ & $10(31)$ & \\
\hline Chemotherapy cycles & & & 1.000 \\
\hline $1-2$ & $24(38)$ & $12(38)$ & \\
\hline $3-5$ & $40(62)$ & $20(62)$ & \\
\hline Type of surgery & & & 0.047 \\
\hline APR & $17(27)$ & $15(47)$ & \\
\hline Sphincter surgery & $47(73)$ & $17(53)$ & \\
\hline
\end{tabular}

Values are presented as mean \pm standard deviation or number (\%). $S C R T$, short-course radiation therapy; $A V$, anal verge; $R T$, radiotherapy; CAPEOX, capecitabine + oxaliplatin; APR, abdominoperineal resection.

weeks in 44 cases (46\%), and was more than or equal to 8 weeks in 52 cases (54\%). There was no difference regarding the time interval between older and younger patients.

\section{Response rates}

Overall, partial and complete tumor response was seen in 82 patients (85.4\%). Evaluation of the primary outcome of the study showed that 20 patients (20.8\%) achieved pCR, 62 cases (64.6\%) partial pathologic response, and 14 patients (14.6\%) were nonresponsive. Table 2 summarizes the pathological response following surgery. There was no statistically significant difference between
Table 2. Distribution and comparison of pathologic characteristics among the older and younger patients with locally advanced rectal cancer treated by neoadjuvant SCRT and chemotherapy followed by delayed surgery

\begin{tabular}{|c|c|c|c|}
\hline \multirow{2}{*}{ Characteristic } & \multicolumn{2}{|c|}{ Age group } & \multirow{2}{*}{$p$-value } \\
\hline & $<65 \mathrm{yr}$ & $\geq 65 \mathrm{yr}$ & \\
\hline pTumor size $(\mathrm{cm})$ & $2.2 \pm 2.2$ & $2.4 \pm 2.0$ & 0.642 \\
\hline pTstage & & & 0.487 \\
\hline T0-2 & $43(67)$ & $24(75)$ & \\
\hline T3-4 & $21(33)$ & $8(25)$ & \\
\hline pNstage & & & 0.270 \\
\hline No & $35(55)$ & $22(69)$ & \\
\hline N1-2 & $29(45)$ & $10(31)$ & \\
\hline $\mathrm{pCR}$ & & & 0.795 \\
\hline Yes & $14(22)$ & $6(19)$ & \\
\hline No & $50(78)$ & $26(81)$ & \\
\hline TRG & & & 0.890 \\
\hline 0 & $14(22)$ & $6(19)$ & \\
\hline 1 & $4(6)$ & $2(6)$ & \\
\hline 2 & $35(55)$ & $20(62)$ & \\
\hline 3 & $11(17)$ & $4(13)$ & \\
\hline Downstaging & & & 0.372 \\
\hline Yes & $52(81)$ & $29(91)$ & \\
\hline No & $12(19)$ & $3(9)$ & \\
\hline Tumor grade & & & 0.824 \\
\hline I & $40(63)$ & $18(56)$ & \\
\hline ॥ & $20(31)$ & $12(38)$ & \\
\hline III & $4(6)$ & $2(6)$ & \\
\hline Surgical margin status & & & 0.683 \\
\hline Involved & $4(6)$ & $3(9)$ & \\
\hline Free & $60(94)$ & $29(91)$ & \\
\hline LVI & & & 0.454 \\
\hline Present & $18(28)$ & $6(19)$ & \\
\hline Absent & $46(72)$ & $26(81)$ & \\
\hline PNI & & & 0.080 \\
\hline Present & $14(22)$ & $2(6)$ & \\
\hline Absent & $50(78)$ & $30(94)$ & \\
\hline KRAS status & & & 0.617 \\
\hline Mutated & $13(20)$ & $8(25)$ & \\
\hline Wild type & $5(8)$ & $1(3)$ & \\
\hline Unknown & $46(72)$ & $23(72)$ & \\
\hline
\end{tabular}

Values are presented as mean \pm standard deviation or number (\%).

$S C R T$, short-course radiation therapy; $p C R$, pathologic complete response; TRG, tumor regression grade; LVI, lymphovascular invasion; PNI, perineural invasion.

the younger and older patients in terms of pCR rate (18.7\% vs. $21.8 \% ; p=0.795)$ and as well as other pathologic characteristics (Table 2). However, the lack of lymphovascular invasion ( $p=$ $0.020)$, lower tumor grade ( $p=0.036)$, larger tumor size $(p=$ 0.005), and the time interval more than 8 weeks between neoadju- 
Table 3. Correlation of $\mathrm{pCR}$ rates and clinical characteristics and the treatments

\begin{tabular}{|c|c|c|c|}
\hline \multirow{2}{*}{ Characteristic } & \multicolumn{2}{|c|}{$\mathrm{pCR}$} & \multirow{2}{*}{$p$-value } \\
\hline & Yes & No & \\
\hline Age (yr) & & & 0.795 \\
\hline$<65$ & $14(22)$ & $50(78)$ & \\
\hline$\geq 65$ & $6(19)$ & $26(81)$ & \\
\hline Sex & & & 0.795 \\
\hline Male & $13(22)$ & 47 (78) & \\
\hline Female & 7 (19) & $29(81)$ & \\
\hline Distance from AV $(\mathrm{cm})$ & $5.9 \pm 2.9$ & $6.5 \pm 3.8$ & 0.546 \\
\hline cTumor size (cm) & $3.7 \pm 1.4$ & $5.3 \pm 2.3$ & 0.005 \\
\hline cTstage & & & 0.059 \\
\hline $\mathrm{T} 2$ & $8(40)$ & $12(60)$ & \\
\hline T3 & $10(15)$ & $55(85)$ & \\
\hline T4 & $2(18)$ & $9(82)$ & \\
\hline cNstage & & & 0.267 \\
\hline No & $3(27)$ & $8(73)$ & \\
\hline $\mathrm{N} 1$ & $13(25)$ & $38(75)$ & \\
\hline N2 & $4(12)$ & $30(88)$ & \\
\hline cStage & & & 0.747 \\
\hline$\|$ & $3(27)$ & $8(73)$ & \\
\hline III & $17(20)$ & $68(80)$ & \\
\hline LVI & & & 0.020 \\
\hline Yes & $1(5)$ & 23 (95) & \\
\hline No & $19(26)$ & $53(74)$ & \\
\hline PNI & & & 0.369 \\
\hline Yes & $2(12)$ & $14(88)$ & \\
\hline No & $18(22)$ & $62(78)$ & \\
\hline Tumor grade & & & 0.036 \\
\hline I & $17(29)$ & 41 (71) & \\
\hline ॥ & $3(9)$ & $29(91)$ & \\
\hline III & $0(0)$ & $6(100)$ & \\
\hline \multicolumn{4}{|l|}{ Chemotherapy regimen } \\
\hline CAPEOX & $16(21)$ & $61(79)$ & 0.979 \\
\hline Capecitabine & $4(21)$ & $15(79)$ & \\
\hline Chemotherapy cycles & & & 0.795 \\
\hline $1-2$ & $7(19)$ & $29(81)$ & \\
\hline $3-5$ & $13(22)$ & 47 (78) & \\
\hline $\begin{array}{l}\text { Time interval between RT and } \\
\text { surgery (wk) }\end{array}$ & & & 0.049 \\
\hline$<8$ & $5(12)$ & $38(88)$ & \\
\hline$\geq 8$ & $15(28)$ & $38(72)$ & \\
\hline
\end{tabular}

Values are presented as number (\%) or mean \pm standard deviation.

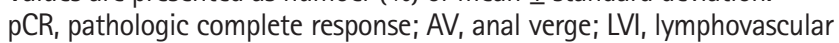
invasion; PNI, perineural invasion; CAPEOX, capecitabine + oxaliplatin; $\mathrm{RT}$, radiotherapy.

vant SCRT and surgery $(p=0.049)$ was correlated with higher rate of pCR (Table 3 ).
Table 4. Frequency severity of treatment-related toxicity in patients with rectal cancer underwent SCRT and chemotherapy

\begin{tabular}{|c|c|c|c|}
\hline \multirow{2}{*}{ Complications } & \multicolumn{2}{|c|}{ Age group } & \multirow{2}{*}{$\mathrm{p}$-value } \\
\hline & $<65 \mathrm{yr}$ & $\geq 65 \mathrm{yr}$ & \\
\hline Diarrhea & & & 0.833 \\
\hline Grade $1-2$ & 55 & 28 & \\
\hline Grade 3-4 & 9 & 4 & \\
\hline Proctitis & & & 0.864 \\
\hline Grade 1-2 & 49 & 25 & \\
\hline Grade 3-4 & 15 & 7 & \\
\hline Noninfectious cystitis & & & 0.794 \\
\hline Grade $1-2$ & 59 & 29 & \\
\hline Grade $3-4$ & 5 & 3 & \\
\hline Neutropenia & & & 1.000 \\
\hline Grade 1-2 & 57 & 29 & \\
\hline Grade $3-4$ & 7 & 3 & \\
\hline Anemia & & & 0.778 \\
\hline Grade $1-2$ & 50 & 25 & \\
\hline Grade 3-4 & 14 & 6 & \\
\hline Thrombocytopenia & & & 0.833 \\
\hline Grade 1-2 & 55 & 28 & \\
\hline Grade 3-4 & 9 & 4 & \\
\hline
\end{tabular}

SCRT, short-course radiation therapy.

\section{Adverse events}

There was no statistically significance in terms of treatment-related side effects including gastrointestinal, urinary and hematologic toxicities between older patients and younger ones (Table 4).

\section{Discussion and Conclusion}

Despite the decline in the incidence and mortality of colorectal cancer in the last decade, it accounts the second leading cause of death in older patients. Rectal cancer consists of about 30\%-40\% of all colorectal cancers [20]. Rectal cancer in older patient tends to be missed and diagnosed in locally advanced stage, particularly in developing countries $[8,9]$. Given the higher rate of underling comorbidities, lower performance status and lower compliance to the treatment, older patients with rectal cancer are frequently undertreated. Therefore, most older patients are spared from many clinical trials and neoadjuvant chemotherapy, and only be treated by SCRT or long-course chemoradiation alone. This modified neoadjuvant therapies may cause lower rate of $\mathrm{pCR}$, as well lower survival rates [10].

In the current clinical trial, we hypnotized that the adding sequential chemotherapy with CAPEOX regimen to SCRT is feasible and tolerable in older patients compared to younger ones. The majority (69\%) of older patients received and tolerated well a median 
three cycles of CAPEOX with acceptable toxicity. In addition, another advantage of CAPEOX regimen, particularly in the COVID-19 era is outpatient administration, reduced hospital visit and limited exposure to the coronavirus that may decline the morbidity and mortality related to COVID-19 infection. According to the ESMO guideline, capecitabine alone or combination of capecitabine and oxaliplatin (such as CAPEOX) is suggested as treatment adapted recommendation in the COVID-19 era for the stage III rectal cancer [11].

In the present study, we found a comparable $\mathrm{pCR}$, downstaging rate, $\mathrm{R} 0$ resection, and treatment-related toxicity in older patients compared to younger patients; however, sphincter-sparing surgery was more frequent among younger patients compared to the older patients. The reason of higher rate of APR in older patient was more due to the higher rate of impaired anal sphincter in tonometry or fecal incontinency at diagnosis, as well the patient and the surgeon preference rather than a surgical technique problem. It is believed that older patients have lower quality of life after LAR due to fecal incontinency and higher rate of LAR syndrome compared to younger patients [21]. LAR syndrome has been poorly defined as a set of complaints, including fecal frequency, urgency, and incontinency, or a feeling of rectal fullness despite defecation. This syndrome causes a considerable impact on the patient's quality of life and leads many patients to select a permanent colostomy to prevent these symptoms [22]. In agreement with our results, Partl et al. [23] and Temple et al. [24] found age as an independent predictive factor for sphincter-preserving surgery following neoadjuvant treatments in patients with rectal cancer. They found a higher rate of sphincter-preserving surgery in younger patients compared to older ones.

In the current study, the rate of pCR was $20.8 \%$ (20 of 96) for all enrolled patients. This $\mathrm{pCR}$ rate is compatible with the results of a systematic review and meta-analysis in which the estimated pooled pCR rate was 17.5\% (95\% confidence interval, 15.7-19.4) in long-course chemoradiation studies; as well as, 11.8\% in SCRT with delay surgery in in the randomized Stockholm III trial $[25,26]$. In the present study, we found a significantly higher rate of $\mathrm{pCR}$ in patients who underwent surgery with a delay time of at least 8 weeks; therefore, it seems that the time interval plays a crucial role in the rate of $\mathrm{pCR}$ to neoadjuvant SCRT and this effect will be more remarkable compared to early surgery. Our finding is in consistent with the several reports and systematic review that confirmed the impact of time interval between SCRT, as well as chemoradiation and surgery on the rate of pCR [12]. Previous studies evaluated and confirmed the efficacy and safety of adding induction chemotherapy to chemoradiation in locally advanced rectal cancer [27]; however, there is paucity and very limited data in the literature regard- ing the feasibility and the safety of neoadjuvant chemotherapy combined with SCRT in older patients with rectal cancer [28]. In a phase II clinical study, Aghili et al. [27] showed that the adding concurrent chemotherapy to SCRT and with delay surgery can provide an enhanced pCR (30.8\%) with acceptable and manageable toxicity. In another randomized controlled trial, they also showed this treatment strategy could provide similar $\mathrm{pCR}$ rate compared to long course chemoradiation [9]. However, a phase 2 multicenter study found short-course concurrent CRT followed by delayed surgery in patients with rectal cancer was associated with poor pCR associated with significant toxicity despite using advanced radiotherapy technique [29]. In a large multicenter randomized clinical trial (RAPIDO), sequential neoadjuvant SCRT and chemotherapy followed by surgery was associated lower disease-related treatment failure compared to standard neoadjuvant long-course chemoradiation followed by surgery followed by adjuvant chemotherapy [30]. In the current study, we used SCRT with sequential chemotherapy rather than concurrent chemotherapy. This treatment strategy was less toxic particularly in older patients. In addition, by this treatment strategy, we achieved a good pCR and acceptable toxicity comparable with previous reports in which patients were treated by combined neoadjuvant chemotherapy and chemoradiation or SCRT [30].

In this clinical trial, we used three-dimensional conformal radiotherapy for delivering SCRT; therefore, by using new radiotherapy techniques such as intensity-modulated radiotherapy that provide radiation dose escalation, more improvement in the $\mathrm{pCR}$ will be expected.

Facing the COVID-19 pandemic was the most important limitation of the study. Continuation of the study based on the initially designed protocol, particularly potentially life-threatening chemotherapy regimen that could decrease immunity and increase the risk of infection was the main challenging issue in elderly patients. Therefore, we had to change the initial chemotherapy regimen (CAPEOX) to capecitabine alone to decrease social exposure of some older patients.

In conclusion, neoadjuvant SCRT and sequential chemotherapy followed by delayed surgery was safe and effective in older patients with locally advanced rectal cancer. A prospective randomized study should be performed to confirm this hypothesis.

\section{Conflict of Interest}

No potential conflict of interest relevant to this article was reported. 


\section{Acknowledgments}

This clinical trial was approved and supported by Shiraz University of Medical Sciences. The manuscript is part of a thesis by Ali Akbar Hafezi.

\section{References}

1. Binefa G, Rodriguez-Moranta F, Teule A, Medina-Hayas M. Colorectal cancer: from prevention to personalized medicine. World J Gastroenterol 2014;20:6786-808.

2. Walker AS, Johnson EK, Maykel JA, et al. Future directions for the early detection of colorectal cancer recurrence. J Cancer 2014;5:272-80.

3. Cassidy RJ, Liu Y, Patel K, et al. Can we eliminate neoadjuvant chemoradiotherapy in favor of neoadjuvant multiagent chemotherapy for select stage II/III rectal adenocarcinomas: analysis of the National Cancer Data base. Cancer 2017;123:783-93.

4. Cameron MG, Kersten C, Vistad I, Fossa S, Guren MG. Palliative pelvic radiotherapy of symptomatic incurable rectal cancer: a systematic review. Acta Oncol 2014;53:164-73.

5. Wu H, Fang C, Huang $L$, et al. Short-course radiotherapy with immediate or delayed surgery in rectal cancer: a meta-analysis. Int J Surg 2018;56:195-202.

6. Zorcolo L, Rosman AS, Restivo A, et al. Complete pathologic response after combined modality treatment for rectal cancer and long-term survival: a meta-analysis. Ann Surg Oncol 2012;19: 2822-32.

7. Koeberle D, Burkhard R, von Moos R, et al. Phase II study of capecitabine and oxaliplatin given prior to and concurrently with preoperative pelvic radiotherapy in patients with locally advanced rectal cancer. Br J Cancer 2008;98:1204-9.

8. van der Geest LG, Elferink MA, Steup WH, et al. Guidelines-based diagnostic process does increase hospital delay in a cohort of colorectal cancer patients: a population-based study. Eur J Cancer Prev 2014;23:344-52.

9. Aghili M, Khalili N, Khalili N, et al. Short-course versus longcourse neoadjuvant chemoradiotherapy in patients with rectal cancer: preliminary results of a randomized controlled trial. Radiat Oncol J 2020;38:119-28.

10. Chang GJ, Skibber JM, Feig BW, Rodriguez-Bigas M. Are we undertreating rectal cancer in the elderly? An epidemiologic study. Ann Surg 2007;246:215-21.

11. Vecchione L, Stintzing S, Pentheroudakis G, Douillard JY, Lordick F. ESMO management and treatment adapted recommendations in the COVID-19 era: colorectal cancer. ESMO Open 2020;5(Suppl 3):e000826.
12. Liu SX, Zhou ZR, Chen LX, Yang YJ, Hu ZD, Zhang TS. Short-course versus long-course preoperative radiotherapy plus delayed surgery in the treatment of rectal cancer: a meta-analysis. Asian Pac J Cancer Prev 2015;16:5755-62.

13. Nguyen NP, Vinh-Hung $V$, Baumert $B$, et al. Older cancer patients during the COVID-19 epidemic: practice proposal of the International Geriatric Radiotherapy Group. Cancers (Basel) 2020;12: 1287.

14. Nasrolahi H, Mirzaei S, Mohammadianpanah M, et al. Efficacy and feasibility of adding induction chemotherapy to neoadjuvant chemoradiation in locally advanced rectal cancer: a phase ii clinical trial. Ann Coloproctol 2019;35:242-8.

15. Myerson RJ, Garofalo MC, El Naqa I, et al. Elective clinical target volumes for conformal therapy in anorectal cancer: a radiation therapy oncology group consensus panel contouring atlas. Int J Radiat Oncol Biol Phys 2009;74:824-30.

16. Treder M, Vogelsang RP, Janssen S, Schild SE, Hollander NH, Rades D. Potential prognostic factors of downstaging following preoperative chemoradiation for high rectal cancer. In Vivo 2018; 32:1481-4.

17. Zhang LN, Xiao WW, Xi SY, et al. Pathological assessment of the AJCC tumor regression grading system after preoperative chemoradiotherapy for Chinese locally advanced rectal cancer. Medicine (Baltimore) 2016;95:e2272.

18. Khan I, Sarker SJ, Hackshaw A. Smaller sample sizes for phase II trials based on exact tests with actual error rates by trading-off their nominal levels of significance and power. $\mathrm{Br} \mathrm{J}$ Cancer 2012;107:1801-9.

19. Faria S, Kopek N, Hijal T, et al. Phase II trial of short-course radiotherapy followed by delayed surgery for locoregionally advanced rectal cancer. Colorectal Dis 2014;16:066-70.

20. Siegel RL, Miller KD, Jemal A. Cancer statistics, 2016. CA Cancer J Clin 2016;66:7-30.

21. Kim BC. Quality of life after a low anterior resection in elderly patients. Ann Coloproctol 2016;32:5-6.

22. Ridolfi TJ, Berger N, Ludwig KA. Low anterior resection syndrome: current management and future directions. Clin Colon Rectal Surg 2016;29:239-45

23. Partl R, Magyar M, Hassler E, Langsenlehner T, Kapp KS. Clinical parameters predictive for sphincter-preserving surgery and prognostic outcome in patients with locally advanced low rectal cancer. Radiat Oncol 2020;15:99.

24. Temple LK, Romanus D, Niland J, et al. Factors associated with sphincter-preserving surgery for rectal cancer at national comprehensive cancer network centers. Ann Surg 2009;250:260-7.

25. Teo MT, McParland L, Appelt AL, Sebag-Montefiore D. Phase 2 neoadjuvant treatment intensification trials in rectal cancer: a 
systematic review. Int J Radiat Oncol Biol Phys 2018;100:14658.

26. Pettersson D, Lorinc $E_{1}$ Holm $T$, et al. Tumour regression in the randomized Stockholm III Trial of radiotherapy regimens for rectal cancer. Br J Surg 2015;102:972-8.

27. Aghili M, Sotoudeh S, Ghalehtaki R, et al. Preoperative short course radiotherapy with concurrent and consolidation chemotherapies followed by delayed surgery in locally advanced rectal cancer: preliminary results. Radiat Oncol J 2018;36:17-24.

28. Sanoff HK, Goldberg RM. Colorectal cancer treatment in older patients. Gastrointest Cancer Res 2007;1:248-53.
29. Yeo SG, Oh JH, Kim DY, et al. Preoperative short-course concurrent chemoradiation therapy followed by delayed surgery for locally advanced rectal cancer: a phase 2 multicenter study (KROG 10-01). Int J Radiat Oncol Biol Phys 2013;86:34-9.

30. Bahadoer RR, Dijkstra EA, van Etten $B$, et al. Short-course radiotherapy followed by chemotherapy before total mesorectal excision (TME) versus preoperative chemoradiotherapy, TME, and optional adjuvant chemotherapy in locally advanced rectal cancer (RAPIDO): a randomised, open-label, phase 3 trial. Lancet Oncol 2021;22:29-42. 